\title{
Direct cloning and transplanting of large DNA fragments from Escherichia coli chromosome
}

\author{
Ying Zhu ${ }^{1,2}$, Yan Yang ${ }^{1,2}$, Pingping Den ${ }^{1,3}$, Yong Huang ${ }^{4}$, Mengxiang $\mathrm{Ni}^{2^{*}}$ \\ \& Hongqing Fang ${ }^{1,3 * *}$ \\ ${ }^{1}$ Institute of Biotechnology, Academy of Military Medical Sciences, Beijing 100071, China; \\ ${ }^{2}$ Institute of Life Science and technology, China Pharmaceutical University, Nanjing 210009, China; \\ ${ }^{3}$ Institute of Health Sciences, Anhui University, Hefei 230039, China; \\ ${ }^{4}$ Institute of Microbiology and Epidemiology, Academy of Military Medical Sciences, Beijing 100071, China
}

Received March 14, 2016; accepted April 26, 2016

\begin{abstract}
We applied a resistance split-fusion strategy to increase the in vivo direct cloning efficiency mediated by Red recombination. The cat cassette was divided into two parts: $\mathrm{cma}$ (which has a homologous sequence with $\mathrm{cmb}$ ) and $\mathrm{cmb}$, each of which has no resistance separately unless the two parts are fused together. The $\mathrm{cmb}$ sequence was integrated into one flank of a target cloning region in the chromosome, and a linear vector containing the cma sequence was electroporated into the cells to directly capture the target region. Based on this strategy, we successfully cloned an approximately $48 \mathrm{~kb}$ DNA fragment from the $E$. coli $\mathrm{DH} 1-\mathrm{Z}$ chromosome with a positive frequency of approximately $80 \%$. Combined with double-strand breakage-stimulated homologous recombination, we applied this strategy to successfully replace the corresponding region of the E. coli DH36 chromosome and knock out four non-essential genomic regions in one step. This strategy could provide a powerful tool for the heterologous expression of microbial natural product biosynthetic pathways for genome assembly and for the functional study of DNA sequences dozens of kilobases in length.
\end{abstract}

Red homologous recombination, resistance split-fusion, target cloning, transferring

Citation: Zhu, Y., Yang, Y., Den, P., Yong, H., Ni, M., and Fang, H. (2016). Direct cloning and transplanting of large DNA fragments from Escherichia coli chromosome. Sci China Life Sci 59, 1034-1041. doi: 10.1007/s11427-016-5100-z

\section{INTRODUCTION}

Red homologous recombination has been widely used in gene knockout, mutation and replacement strategies in molecular biology research (Datsenko and Wanner, 2000; Ellis et al., 2001; Murphy, 1998; Muyrers et al., 2000; Yu et al., 2000). Target DNA can be directly cloned into the cloning vector mediated by the Red recombinant technique based on the interaction between homologous sequences in vivo (Bian et al., 2012; Cobb and Zhao, 2012; Fu et al., 2012; Zhang et al., 2000). Depending on whether the target DNA is cir-

*Corresponding author (email: Nimx_2000@aliyun.com)

**Corresponding author (email: fanghongqing@vip.sina.com; fanghq@nic.bmi.ac) cular or linear, the direct cloning method can be divided into two categories (Fu et al., 2012): LCHR (linear plus circular homologous recombination) and LLHR (linear plus linear homologous recombination). Target DNA regions of approximately $30 \mathrm{~kb}, 3 \mathrm{~kb}$ fragments from plasmid BACs (bacterial artificial chromosomes) and genomic DNA fragments can be easily cloned by LCHR assay (Zhang et al., 2000). Due to the complexity of the DNA sequence, it is almost impossible to obtain positive clones using conventional methods when the length of the target DNA is more than $10 \mathrm{~kb}$.

In order to directly clone a target DNA sequence of dozens of kilobases in length from the bacterial chromosome, a resistance split-fusion strategy was applied (Figure 1). The 


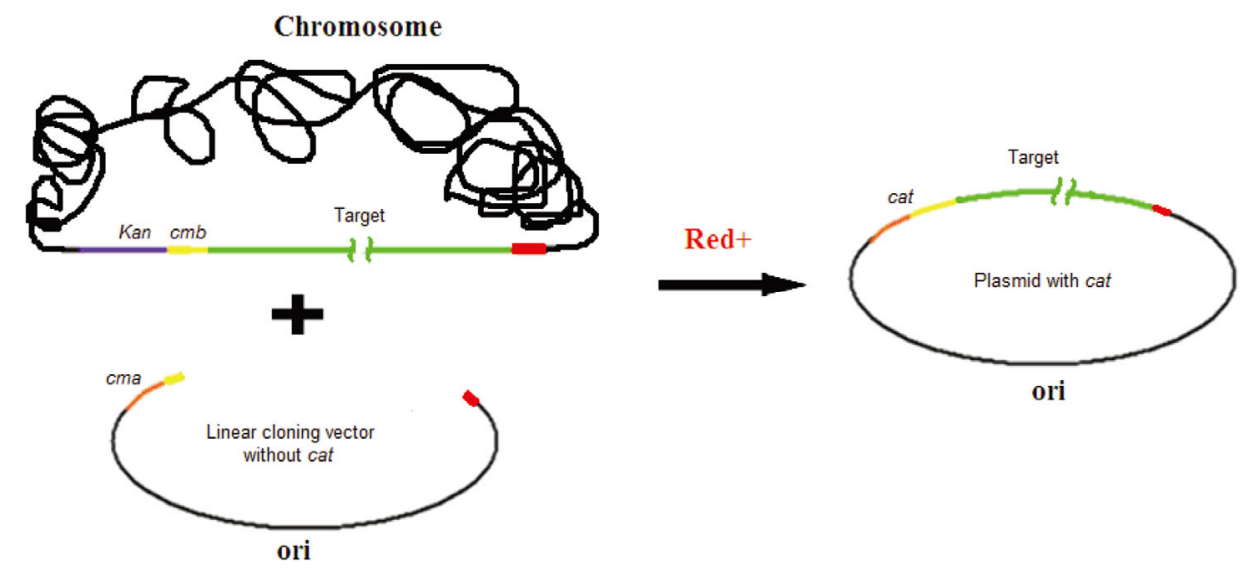

Figure 1 The principle of the resistance split-fusion strategy for direct cloning DNA sequence.

cat cassette harbored in linear plasmid was divided into two parts: cma (which shares a homologous sequence, $135 \mathrm{bp}$ in length, with $\mathrm{cmb}$ ) and $\mathrm{cmb}$, each of which has no resistance separately unless the two parts are fused together. First, we directionally integrated the $c m b$ sequence and a full-length kanamycin-resistance gene (as the marker for this step) into a flanking region adjacent to the target region on the chromosome. Next, a linear vector containing the cma sequence was electroporated into the cells; this vector also contained a $50 \mathrm{bp}$ sequence with homology to the other flank of the target region. The $\mathrm{cmb}$ sequence adjacent to the target region of the chromosome and the cma sequence in the linear cloning vector were fused to form a complete cat gene. With the aid of ampicillin resistance screening, the direct cloning efficiency was greatly improved. Based on this strategy, we successfully cloned an approximately $48.2 \mathrm{~kb}$ DNA fragment named "unit Z" from the E. coli DH1-Z chromosome (unit $\mathrm{Z}$ has four markerless-deleted nonessential genomic regions). Then, the recombinant plasmid containing unit $\mathrm{Z}$ was introduced into DH36 cells. Combined with double-strand breakage-stimulated homologous recombination, the corresponding region of the $E$. coli DH36 chromosome was successfully replaced by unit $\mathrm{Z}$ to produce an E. coli strain DH40 with four nonessential genomic regions knocked out in one step. This strategy can be used in genome assembly and the functional study of DNA sequences dozens of kilobases in length.

\section{RESULTS}

\section{Direct cloning and identification of unit $\mathbf{Z}$}

To further reduce the $E$. coli $\mathrm{DH} 36$ chromosome by replacing the corresponding region with unit $\mathrm{Z}$ after deleting four nonessential genomic regions, the deleted unit $\mathrm{Z}$ (hereinafter referred to as unit $Z$ ) should be directly captured first. Unit $\mathrm{Z}$ is approximately $48 \mathrm{~kb}$ in length and could not be captured by general Red reconstruction method (Zhang et al., 2000), but was successfully cloned from E. coli DH1-Z chromosome by resistance split-fusion strategy (Figure 2). When screened by PCR with the primer pairs 38-0 and $\mathrm{AD}-1$, and 35-1 and M13F, the frequency of positive clones was as high as $89 \%(17 / 19)$ (Figure $3 \mathrm{~A}$ and $\mathrm{B}$ ). The recombinant plasmid p15AD2IC-Z was transferred to E. coli DH1 and then extracted, for the E. coli DH1-Z genomic DNA may influence the PCR identification result. To ensure the four non-essential genomic regions were deleted, PCR identification of the recombinant plasmid was performed with the primer pairs ZL-R5 and 35-1, 36-0 and 36-1, 37-0 and 37-1 and 38-0 and 38-1. In addition, three restriction enzymes, Apa I, Sac I, and Xba I were chosen for the restriction digestion analysis of p15AD2IC-Z. Consistent with the predicted pattern, digested p15AD2IC-Z plasmids generated DNA fragments of approximately approximate 17.2,
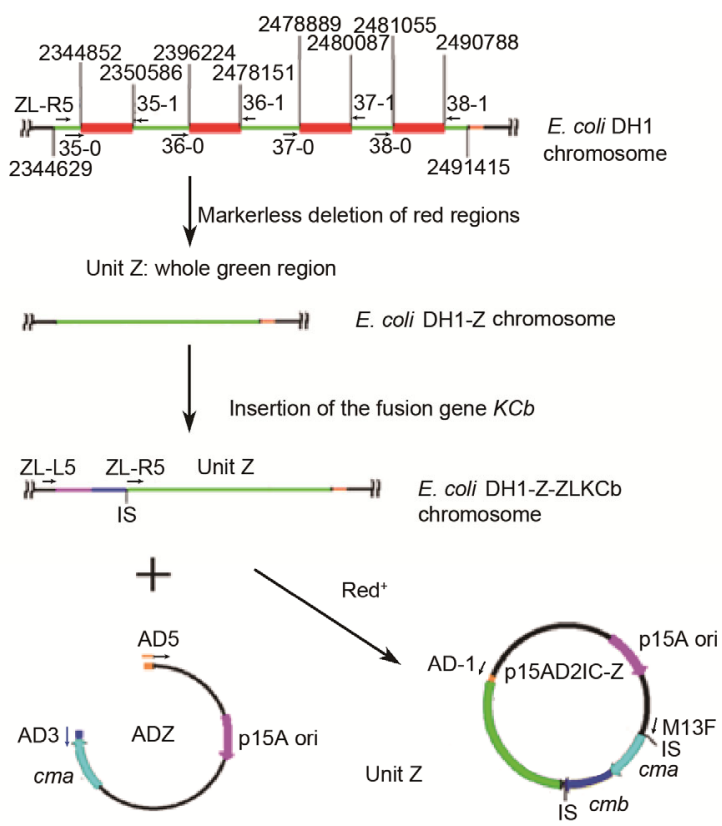

Figure 2 Schematic diagram for cloning unit Z. IS, I-Sce I restriction site. 
14.7, 14.4, 3.3, and 1.4 kb (Figure 4A). The restriction digest showed that the first three bands were too close in size to be separated, although there was a bright and wide band near $15 \mathrm{~kb}$, and bands were visible at 3.3 and $1.4 \mathrm{~kb}$ (Figure 4B). The two identification results above showed that p15AD2IC-Z was the positive recombinant plasmid.

\section{Knocking out four non-essential regions of the receptor chromosome by the replacement of unit $\mathrm{Z}$}

The schematic diagram for the co-transformation of the donor plasmid p15AD2IC-Z and the helper plasmid pAAISGBE into E. coli DH36-35LRIK-38LRIT is shown in Figure 5. The Red recombinase system and I-Sce I endonuclease were induced by arabinose, and after recombination, the cells were plated onto LB plates and cultivated. The positive clone, without kanamycin and ampicillin resistance, was obtained by resistance loss screening.

Further PCR identification of the recombinant strain was performed with primer pairs ZL-L5 and 35-1, 36-0 and $36-1,37-0$ and 37-1, and 38-0 and ZR-R3, and showed that four regions of the positive clone were all $\sim 1.2 \mathrm{~kb}$ in length, as predicted (Figure 6). High fidelity PCR amplification of the sequences 35LR, 36LR, 37LR and 38LR was performed with primer pairs $35-1$ and $35-0,36-1$ and 36-0, 37-1 and $37-0$ and $38-0$ and $38-1$, and the products were sequenced; the sequences obtained were as expected. In addition to the identification of 36 other deleted regions, the recombinant strain E. coli DH40 was confirmed to contain the markerless deletion of four non-essential chromosomal regions.

Furthermore, re-sequencing of genomic DNA was performed on E. coli DH36 and E. coli DH40 strains to confirm the internal sequence of unit $\mathrm{Z}$ had no unexpected recombination; the alignment of the relevant chromosome regions obtained using CLC Genomics Workbench is shown in Figure 7. The blank region of the E. coli DH40 chromosome compared with $E$. coli DH36 were the knocked out regions $35,36,37,38$, and the remainder of the sequence did not recombine unexpectedly. As a result, 40 nonessential regions of the positive recombinant $E$. coli strain DH40 have been knocked out tracelessly, and the genome was reduced by $20.4 \%$ for a total deletion of $945 \mathrm{~kb}$.
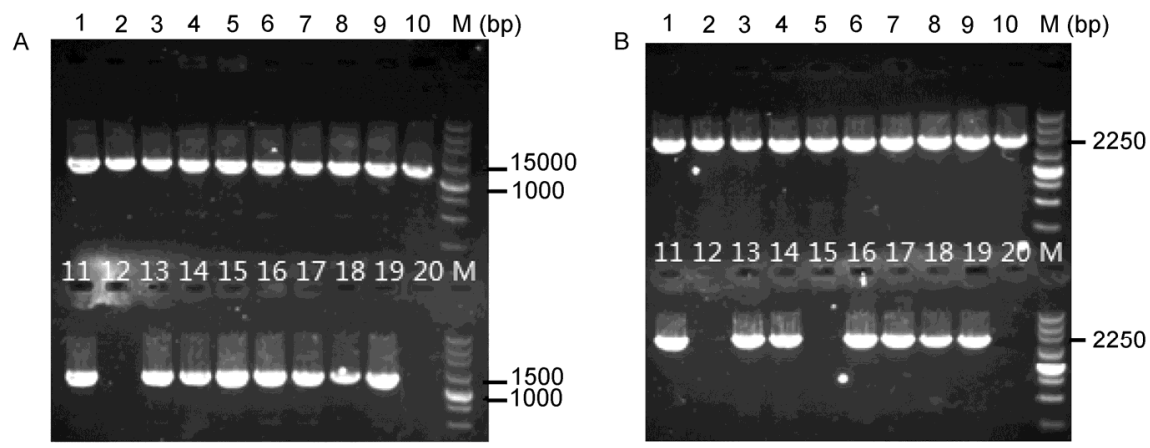

Figure 3 PCR screening for cloning unit Z. A, Primers 38-0 and AD-1 (the positive product is approximately $1.5 \mathrm{~kb}$ ). B, Primers M13F and 35-1 (the positive product is about $2.5 \mathrm{~kb}$ ). $1-19$, sample; 20, p15AD2IC-SacB; M, 250 bp DNA ladder marker.
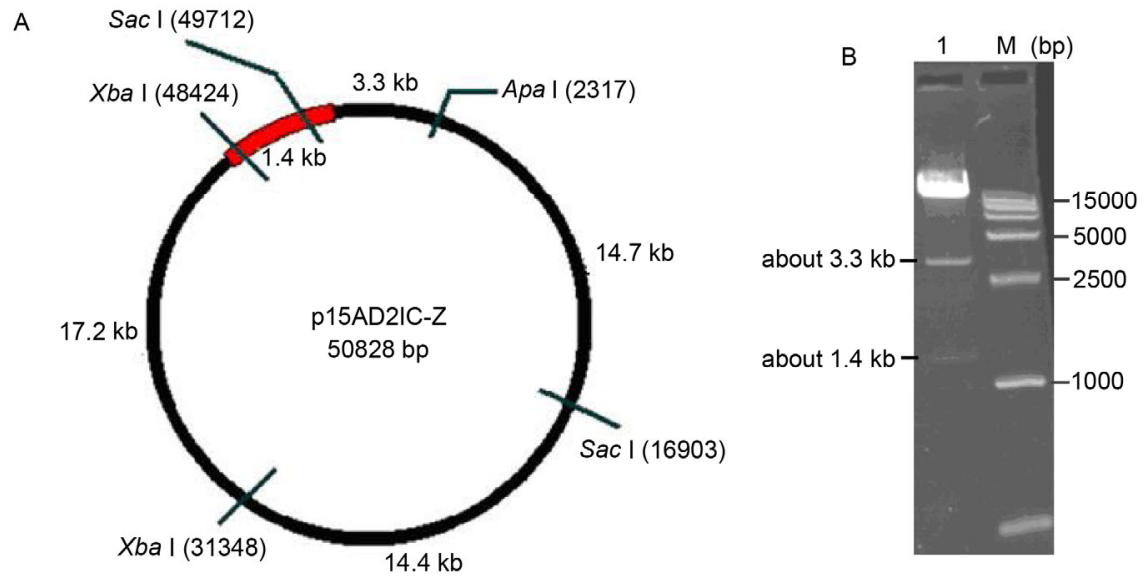

Figure 4 The restriction site of p15AD2IC-Z. A, The restriction site of p15AD2IC-Z (the red region is the cloning vector). B, The digestion analysis of p15AD2IC-Z by restriction enzymes Apa I, Sac I, and Xba I. M, DL 15000 DNA Marker; 1, p15AD2IC-Z. 


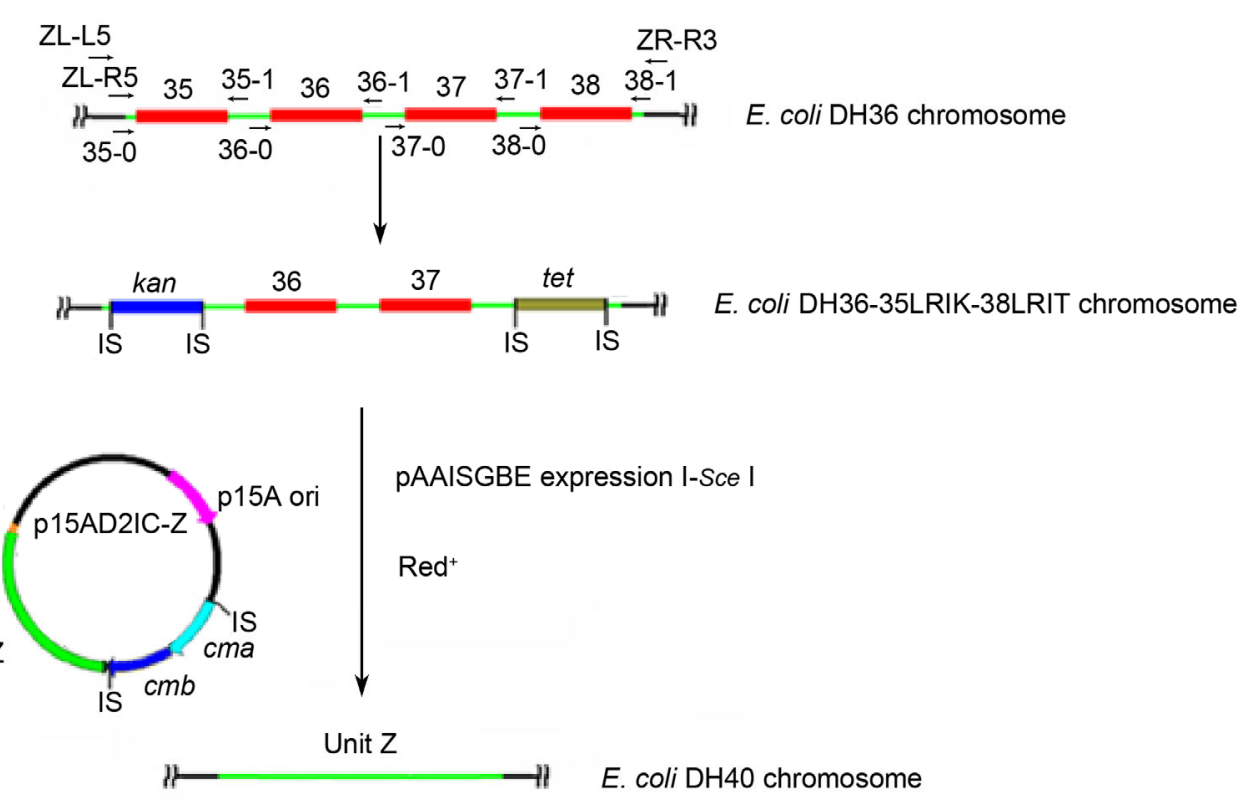

Figure 5 Schematic diagram of transplanting unit Z.

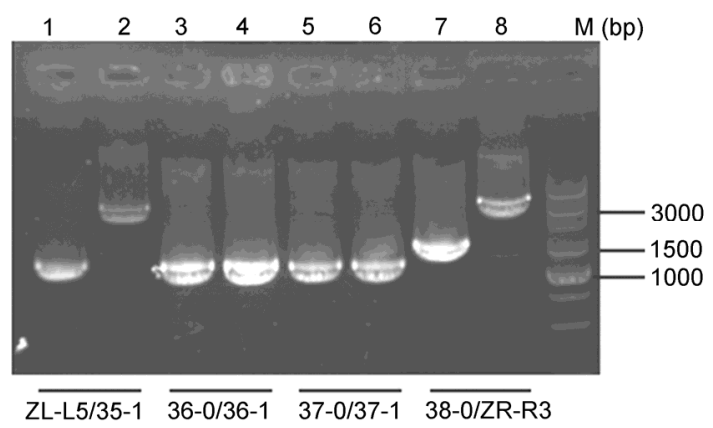

Figure 6 PCR verification of transplantation of unit Z. 1 and 2, Primers ZL-L5/35-1; 3 and 4, Primers 36-0/36-1; 5 and 6, Primers 37-0/37-1; 7 and 8, Primers 38-0/ZR-R3. 1,3,5,7, E. coli DH40; 2,4,6,8, E. coli DH3635LRK-38LRT/p15AD2IC-Z/pAAISGBE. M, 250 bp DNA ladder marker.

\section{DISCUSSION}

Target DNA, dozens of kilobases in length, can be directly cloned from BAC vectors, mediated by the Red recombinant system. However, the positive rate of direct cloning from chromosomes is very low because the sequence and structure of chromosomes are much more complex than that of the BAC vectors (Fu et al., 2012; Zhang et al., 2000). There are two strategies for direct cloning of large DNA sequences: LCHR and LLHR (Fu et al., 2012). Target DNA sequences from genomes or BAC vectors can be directly cloned by LCHR, and can also be digested to fragments containing the target DNA sequence using restriction enzymes and then co-electroporated with linear vectors into E. coli $\mathrm{Red} / \mathrm{ET}^{+}$cells to allow direct cloning of the target sequence via LLHR. The LCHR method can also be applied to the reformation of recombinant plasmids, such as the replacement wild type promoters with synthetic promoters, adding modifier genes and so on; these recombinant plasmids may acquire changes in copy number, expression direction and host range of heterologous expression (Sharan et al., 2009; Venken et al., 2006; Wenzel et al., 2005). However, both of these strategies will produce a large number of empty vectors and recombinants generated by the linear vectors and other nonspecific regions. Therefore, the PCR screening of recombinants can be time-consuming, for false positive clones may also contain the antibiotic resistance marker present in the original vector.

In this study, we improved the conventional Red homologous recombination method using a resistance split-fusion strategy, and combined with the LCHR method, we successfully directly cloned a target DNA sequence, $48.2 \mathrm{~kb}$ in length, from the E. coli DH36-35LRIK-38LRIT chromosome. The positive rate reached $80 \%$, yet the target fragment could not be directly cloned using Red homologous recombination alone.

For practical applications, the different plasmids should be selected according to the size of the target DNA fragments. Studies have found that using high-copy plasmids and medium-copy vectors as the acceptor such as pUC, pBluescript (copy number 500-700), pBR322, p15A, (copy number 15-20), the upper limit length of the target DNA sequence was about 25-80 kb (Venken et al., 2006). When length of the target DNA sequence was hundreds of kilobases, single copy plasmids such as BAC plasmids were required, for BAC plasmids can carry 200-250 kb exogenous DNA fragments, and single-copy plasmids are more stable in host cells (Kotzamanis and Huxley, 2004).

In this study, the resistance split-fusion strategy was designed to increase the direct cloning efficiency based on the Red recombination system. The cat cassette in p15A plas- 
mid was divided into two parts: $\mathrm{cma}$ and $\mathrm{cmb}$, and the $\mathrm{cmb}$ fragment was integrated into chromosome while the cma fragment was retained in a linear plasmid. Each of these two parts has no resistance separately unless they are fused together. Based on this strategy, a target DNA fragment of approximately $48 \mathrm{~kb}$ in length was directly cloned from the E. coli $\mathrm{DH} 1-\mathrm{Z}$ chromosome. E. coli strain DH1-Z was constructed from $E$. coli $\mathrm{DH} 1$ by constructing a markerless knockout of four non-essential genomic regions in unit $\mathrm{Z}$; thus, unit Z of E. coli DH1-Z was reduced from 146.8 to $48.2 \mathrm{~kb}$, and the size of the recombinant plasmid p15AD2IC-Z (which replicated stably in cells) was about $50 \mathrm{~kb}$. Then, combined with double-strand breakagestimulated homologous recombination, the corresponding region of $E$. coli $\mathrm{DH} 36$ chromosome was replaced with unit $\mathrm{Z}$ of $E$. coli $\mathrm{DH} 1-\mathrm{Z}$, and the resulting strain was $E$. coli DH40. In short, the chromosome was decreased in size by about $100 \mathrm{~kb}$ as four non-essential genomic regions were knocked out in one step.

This strategy provides a powerful tool for the heterologous expression of microbial natural product biosynthetic pathways, genome assembly, and the functional study of DNA sequences dozen of kilobases in length. Gene clusters can be directly cloned from unsequenced genomes into expression vectors, or integrated into heterologous chromosomes for heterologous expression to characterize their functions (Ongley et al., 2013a). Based on this, through homologous recombination, the function and regulation of gene clusters can be reconstructed to improve metabolic flux and then improve the expression of natural products. Traditional de novo assembly is not conducive to the assembly of disparate biosynthetic pathways, which are encoded by dozens or even hundreds of kilobases of DNA, but methods of direct cloning can not only obtain target gene clusters and assemble multiple large fragments into large gene cluster families (Kotzamanis and Huxley, 2004), but can also reform them by site-directed mutagenesis, insertion, and markerless deletion. Then, the reformed gene clusters may synthesize derivatives of natural products. Furthermore, direct cloning and heterologous expression of corresponding pathways for the synthesis of natural products from some microorganisms that are difficult to cultivate in the laboratory may have high-yield products with safety and efficiency and save on costs (Ongley et al., 2013b). In conclusion, this cloning method could be used to discover potential large gene clusters and find valuable natural products; on the other hand, it can also be used in reconstructing pathways for heterologous expression and the synthesis of novel derivatives of natural products.

\section{MATERIALS AND METHODS}

\section{Strains, plasmids, primers, enzymes and reagents}

Bacterial strains, plasmids and primers used in this study are shown in Tables 1 and 2 .

Dpn I was purchased from NEB (Ipswich, USA) and other enzymes and DNA markers were purchased from TaKaRa Biotechnology (Dalian). Mini plasmid kits were purchased from TIANGEN BIOTECH (Beijing). PurePlasmid mini kits were purchased from CWBIOTECH (USA). Ampicillin, chloramphenicol, kanamycin, tetracycline were purchased from GENVIEW (USA).

LB medium: peptone (Oxoid, UK) $10 \mathrm{~g} \mathrm{~L}^{-1}$, yeast extract (Oxoid) $5 \mathrm{~g} \mathrm{~L}^{-1}, \mathrm{NaCl} 10 \mathrm{~g} \mathrm{~L}^{-1}$, pH 7.0. LB plates also contained $20 \mathrm{~g} \mathrm{~L}^{-1}$ agar and sucrose LB plates also contained $60 \mathrm{~g} \mathrm{~L}^{-1}$ sucrose, without $\mathrm{NaCl}$. When appropriate, antibiotics were added to the culture medium at the following concentrations: ampicillin $100 \mathrm{mg} \mathrm{L}^{-1}$, chloramphenicol $50 \mathrm{mg} \mathrm{L}^{-1}$, kanamycin $50 \mathrm{mg} \mathrm{L}^{-1}$, tetracycline $10 \mathrm{mg} \mathrm{L}^{-1}$.

\section{Construction of fragment $\mathrm{KCb}$ containing fused $\mathrm{kan}$ and $c m b$}

Fragment $\mathrm{KCb}$ was produced using PCR amplification as follows. PCR amplification of kan was performed with primers K-F (which created a EcoR I restriction site at the 5' end of the amplicon) and K-R, using plasmid pKD4 as the template. PCR amplification of $\mathrm{cmb}$ was performed with primers ICB-F and ICB-R (which created a $B a m \mathrm{H}$ I restriction site in the $3^{\prime}$ end of the amplicon), using plasmid p15AD2IC-SacB as the template. Primers K-F and ICB-R were then used to amplify the fusion PCR product, $K C b$, using the PCR fragments mentioned before as the template. $K C b$ was digested with EcoR I and BamH I and inserted into the EcoR I and BamH I sites of vector pUC19, and the resulting plasmid was named pUC19-KCb.

\section{Direct cloning unit $\mathrm{Z}$ from $E$. coli DH1-Z chromosome by the resistance split-fusion strategy}

Unit Z was a region of the E. coli DH1 chromosome (2344629...2491415), and E. coli DH1-Z was constructed by knocking out four non-essential genomic regions, region 35 (2344852..2350586), region 36 (2396224..2478151), region $37(2478889 . .2480087)$ and region $38(2481055 \ldots$ 2490788) (Figure 2). Unit $\mathrm{Z}$ of the E. coli DH1-Z chromosome was $48.2 \mathrm{~kb}$ in length with a reduction of about 100 kb compared to the E. coli DH1 chromosome. The process of direct cloning unit $\mathrm{Z}$ via the Red homologous recombination system was achieved in two steps:

(i) Fragment $\mathrm{KCb}$ was integrated into the left flank of unit $\mathrm{Z}$ on the E. coli DH1-Z chromosome (2344628... 2344629 ) using a method similar to Gene-Doctoring (Lee et al., 2009); the resulting strain was E. coli DH1-Z-ZLRKCb. PCR amplification of the upstream homologous region ZLL was performed using the primer pair ZL-L5 and ZL-L3, using DH1 chromosome as the template. PCR amplification of the downstream homologous region ZLR was performed using the primer pair ZL-R5 and ZL-R3, using DH1 chromosome as the template. We digested ZLL with EcoR I and 
Table 1 Bacterial strains and plasmids used in this study

\begin{tabular}{|c|c|c|}
\hline Strain or plasmid & Genotype or characteristic & Source or reference \\
\hline \multicolumn{3}{|l|}{ E. coli Strain } \\
\hline BL21(AI) & $F$ - ompT hsdSB $\left(\mathrm{r}_{\mathrm{B}^{-}} \mathrm{m}_{\mathrm{B}^{-}}\right)$gal dcm araB::T7RNAP-tet $A$. To supply Tet $^{\mathrm{r}}$ gene & Invitrogen \\
\hline DH5 $\alpha$ & supE44 lacZAM15 hsdR17 recA1 endA1 gyrA96 thi-1 relA1 & Transgen \\
\hline DH1 & The genomic sequence is listed in Accession no. CP001637 & ATCC 33849 \\
\hline DH36 & Derivative of $E$. coli $\mathrm{DH} 1$ with 36 non-essential regions knocked out & This study \\
\hline DH1-Z & $\begin{array}{l}\text { Derivative of } E \text {. coli DH1 with region } 35 \text {, region } 36 \text {, region } 37 \text {, region } 38 \text { in unit Z knocked out, shown in Fig- } \\
\text { ure } 2\end{array}$ & This study \\
\hline $\begin{array}{l}\text { DH40 } \\
\text { plasmid }\end{array}$ & Derivative of $E$. coli $\mathrm{DH} 36$ with 4 non-essential regions knocked out & This study \\
\hline pKD4 & Conferred kan cassette & $\begin{array}{l}\text { (Datsenko and } \\
\text { Wanner, 2000) }\end{array}$ \\
\hline pAAGBE & $\begin{array}{l}\text { Derivative of pKOBEG (Chaveroche et al., 2000) with cat replaced by amp. A temperature-sensitive helper } \\
\text { plasmid expresses Red recombinase by adding L-arabinose }\end{array}$ & This study \\
\hline pAAISGBE & $\begin{array}{l}\text { A temperature-sensitive helper plasmid expresses I-Sce I endonuclease and Red recombinase by adding } \\
\text { L-arabinose, } a m p\end{array}$ & This study \\
\hline pAAICDGBE & $\begin{array}{l}\text { A temperature-sensitive helper plasmid expresses I-Cre I endonuclease and Red recombinase by adding } \\
\text { L-arabinose, } a m p\end{array}$ & This study \\
\hline p15AD2IC-SacB & $\begin{array}{l}\text { The donor plasmid containing p15A ori, cat resistance gene flanked by I-Cre I cleavage site (named as IC) and } \\
\text { the counter-selection marker } \operatorname{sacB} \text { (a plasmid having } s a c B \text { cannot grow in media containing sucrose) (Li et al., } \\
\text { 2013) }\end{array}$ & This study \\
\hline
\end{tabular}

Table 2 Primers used in this study

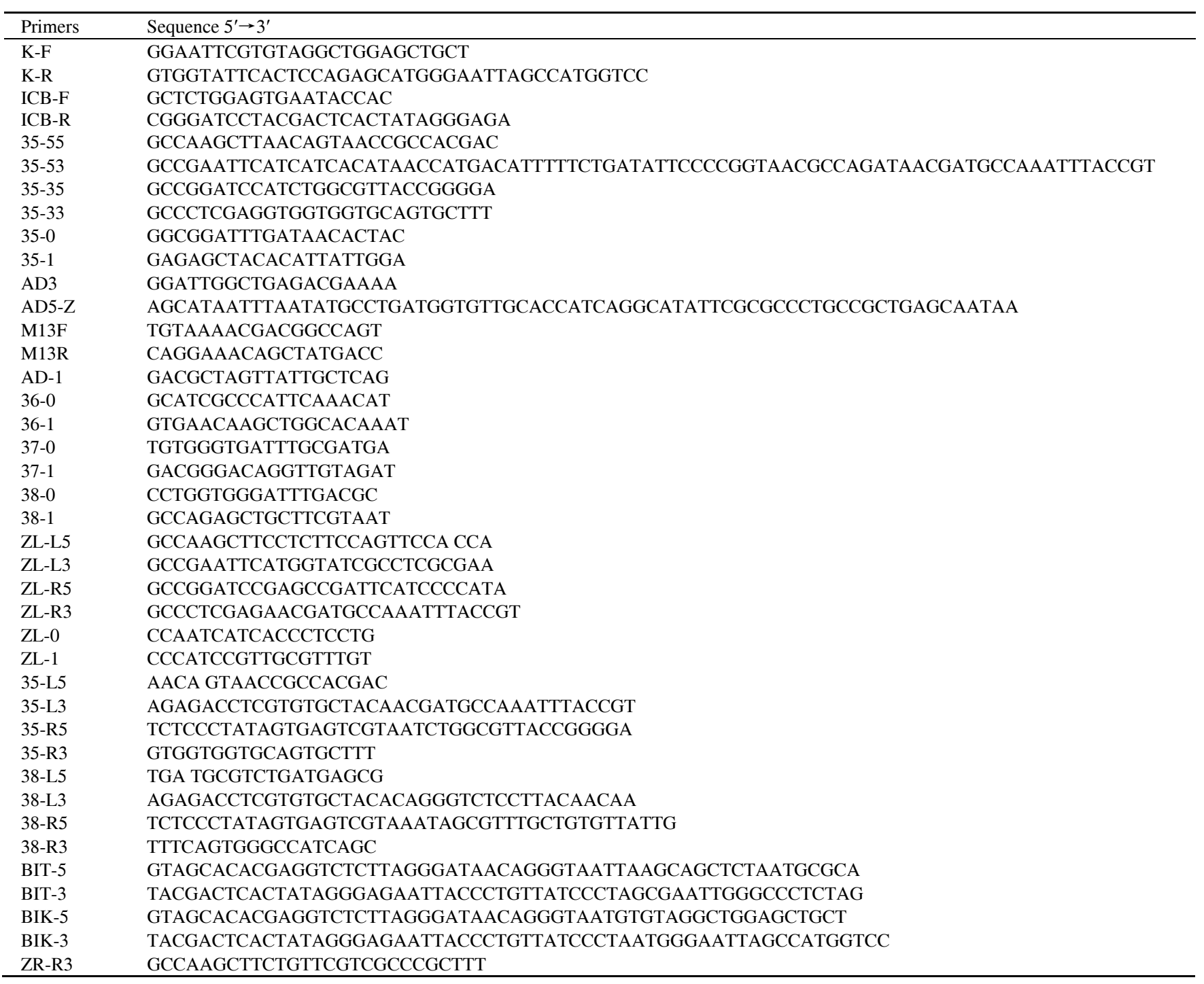




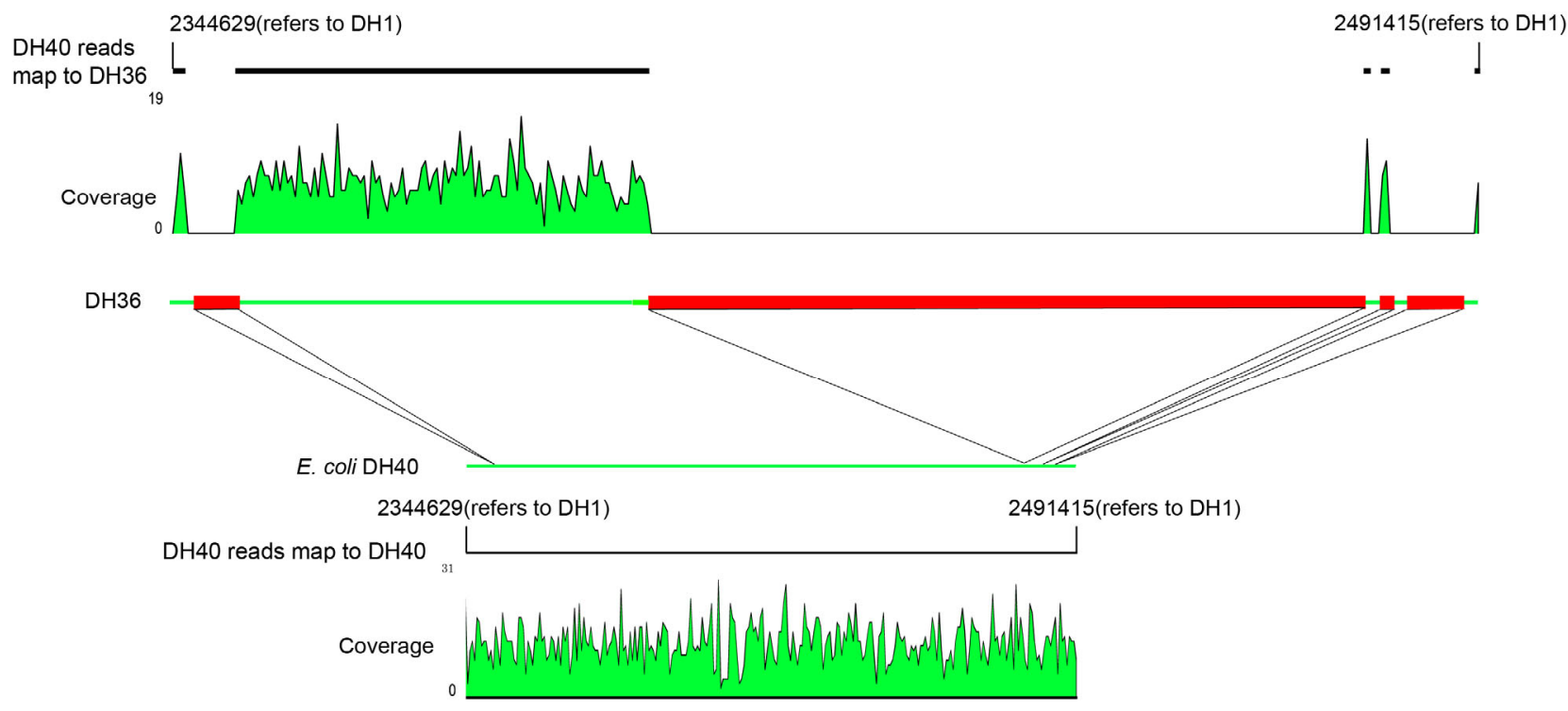

Figure 7 Genome DNA re-sequencing alignment of E. coli DH36 and DH40. Sequence 2344629...2491415 refers to E. coli DH1; red regions were deleted regions.

Hind III, ZLR with BamH I and Xho I, KCb with Eco R I and BamH I and inserted them into Hind III and Xho I sites of vector p15AD2IC-SacB; the resulting plasmid was named p15AD2-ZLLR-KCb-SacB. After double resistance screening (using ampicillin and kanamycin), a single clone of strain E. coli DH1-Z harboring p15AD2-ZLLR-KCbSacB and pAAICDGBE was inoculated into the LB medium with ampicillin and then cultivated at $30^{\circ} \mathrm{C}$ for $4 \mathrm{~h}$ with $0.2 \%$ L-arabinose in medium. 10-fold dilutions of the cells were plated onto LB plates containing kanamycin and sucrose and cultured overnight. The resulting strain $E$. coli DH1-Z-ZLRKCb was verified by PCR identification using the primer pair ZL-0 and ZL-1.

(ii) PCR amplification of the linear plasmid ADZ was carried out using p15AD2IC-SacB (after BamH I digestion) as the template and using the primer pair AD5-Z and AD3. As designed, the ends of ADZ had 135 and 50 bp homologous sequences with $\mathrm{cmb}$ and the chromosome, respectively. The PCR fragment ADZ was electroporated into $E$. coli DH1-Z-ZLKCb harboring pAAGBE, and the recombinant strain was selected for on LB plates containing kanamycin and sucrose. After PCR identification using the primer pairs M13F and 35-1 and 38-0 and AD-1, the positive vector was transferred into E. coli $\mathrm{DH} 1$, and the extracted plasmids were analyzed and identified by restriction digest and PCR; the resulting plasmid was p15AD2IC-Z.

\section{Transplanting of unit $\mathrm{Z}$ to $E$. coli $\mathrm{DH} 36$ chromosome}

It was difficult to achieve a complete replacement of the target region, for sequence of unit $\mathrm{Z}$ in p15AD2IC- $\mathrm{Z}$ was highly homologous with the corresponding region on the $E$. coli DH36 chromosome. Two I-Sce I restriction sites were inserted on both sides of the replacement region of DH36 chromosome, and the corresponding region of approximately $146.8 \mathrm{~kb}$ in length was replaced by unit $\mathrm{Z}(48.2 \mathrm{~kb}$ in length) via a double-strand breakage-stimulated homologous recombination strategy. The schematic diagram of transplanting unit $\mathrm{Z}$ is shown in Figure 5. Fragments 35LR-IK and 38LR-IT were integrated into region 35 and region 38, respectively, then I-Sce I homing endonucleases were used to digest the DNA at the I-Sce I restriction sites in the chromosome. As a result, the double-stranded DNA was cleaved and then repaired by homologous recombination between unit $\mathrm{Z}$ and over $500 \mathrm{bp}$ of homologous sequence of double strand breaks due to the action of Red recombinant enzyme.

Fragments 35LR-IK and 38LR-IT containing the markers $I S$-kan and IS-tet were obtained by overlap extension PCR. PCR amplification of $35 \mathrm{~L}$ and $35 \mathrm{R}$ was performed using the primer pairs $35 \mathrm{~L}-5$ and $35 \mathrm{~L}-3$ and $35 \mathrm{R}-5$ and $35 \mathrm{R}-3$, and using the DH1 chromosome as the template. PCR amplification of IS-kan was performed using the primer pairs BIK-5 and BIK-3 and pKD4 as the template, and then using the primer pairs $35 \mathrm{~L}-5$ and $35 \mathrm{R}-3$ and the PCR fragments mentioned before as template; the resulting PCR fragment was 35LR-IK. Using the same method, fragment 38LR-IT was obtained. Both of these fragments were electroporated into $E$. coli $\mathrm{DH} 36$ and integrated into region 35 and region 38 , and the resulting strain was E. coli DH36-35LRIK38LRIT.

Plasmid p15AD2IC-Z was co-transformed with pAAISGBE into $E$. coli DH36-35LRIK-38LRIT, and then a single clone was inoculated into LB medium containing chloramphenicol and ampicillin and cultured overnight. After that, the cells were cultivated at $30^{\circ} \mathrm{C}$ for $4 \mathrm{~h}$ with $0.2 \% \mathrm{~L}$-arabinose in the medium. Ten-fold dilutions of the 
cells were plated onto LB plates and cultured overnight. $E$. coli strain DH40 was confirmed by screening by resistance loss and PCR identification using the primer pairs ZL-R5 and $35-1,36-0$ and $36-1,37-0$ and $37-1$ and $38-0$, and ZR-R3.

Compliance and ethics The author(s) declare that they have no conflict of interest.

Acknowledgements This work was supported by the National Natural Science Foundation of China (81373286), and National Basic Research Program of China (2011CBA00800).

Bian, X., Huang, F., Stewart, F.A., Xia, L., Zhang, Y., and Müller, R. (2012). Direct cloning, genetic engineering, and heterologous expression of the syringolin biosynthetic gene cluster in $E$. coli through Red/ET recombineering. Chem Bio Chem 13, 1946-1952.

Chaveroche, M.K., Ghigo, J.M., and d'Enfert, C. (2000). A rapid method for efficient gene replacement in the filamentous fungus Aspergillus nidulans. Nucleic Acids Res 28, e97-e97.

Cobb, R.E., and Zhao, H. (2012). Direct cloning of large genomic sequences. Nat Biotechnol 30, 405.

Datsenko, K.A., and Wanner, B.L. (2000). One-step inactivation of chromosomal genes in Escherichia coli K-12 using PCR products. Proc Natl Acad Sci USA 97, 6640-6645.

Ellis, H.M., Yu, D., and DiTizio, T. (2001). High efficiency mutagenesis, repair, and engineering of chromosomal DNA using single-stranded oligonucleotides. Proc Natl Acad Sci USA 98, 6742-6746.

Fu, J., Bian, X., Hu, S., Wang, H., Huang, F., Seibert, P.M., Plaza, A., Xia, L., Müller, R., and Stewart, A.F. (2012). Full-length RecE enhances linear-linear homologous recombination and facilitates direct cloning for bioprospecting. Nat Biotechnol 30, 440-446.

Kotzamanis, G., and Huxley, C. (2004). Recombining overlapping BACs into a single larger BAC. BMC Biotechnol 4, 1.
Lee, D.J., Bingle, L.E., Heurlier, K., Pallen, M.J., Penn, C.W., Busby, S.J., and Hobman, J.L. (2009). Gene doctoring: a method for recombineering in laboratory and pathogenic Escherichia coli strains. BMC Microbiol 9,1 .

Li, X.-T., Thomason, L.C., Sawitzke, J.A., and Costantino, N. (2013). Positive and negative selection using the tetA-SacB cassette: recombineering and P1 transduction in Escherichia coli. Nucleic Acids Res 41, e204-e204.

Murphy, K.C. (1998). Use of bacteriophage $\lambda$ recombination functions to promote gene replacement in Escherichia coli. J Bacteriol 180, 2063-2071.

Muyrers, J.P., Zhang, Y., Buchholz, F., and Stewart, A.F. (2000). $\operatorname{RecE} / \operatorname{Rec} T$ and $\operatorname{Red} \alpha / \operatorname{Red} \beta$ initiate double-stranded break repair by specifically interacting with their respective partners. Genes Dev 14, 1971-1982.

Ongley, S.E., Bian, X., Neilan, B.A., and Müller, R. (2013a). Recent advances in the heterologous expression of microbial natural product biosynthetic pathways. Nat prod Rep 30, 112-1138.

Ongley, S.E., Bian, X., Zhang, Y., Chau, R., Gerwick, W.H., Muiller, R., and Neilan, B.A. (2013b). High-titer heterologous production in E. coli of lyngbyatoxin, a protein kinase $\mathrm{C}$ activator from an uncultured marine cyanobacterium. ACS Chem Biol 8, 1888-1893.

Sharan, S.K., Thomason, L.C., Kuznetsov, S.G., and Court, D.L. (2009). Recombineering: a homologous recombination-based method of genetic engineering. Nat Protoc 4, 206-223.

Venken, K.J., He, Y., Hoskins, R.A., and Bellen, H.J. (2006). P [acman]: a BAC transgenic platform for targeted insertion of large DNA fragments in D. melanogaster. Science 314, 1747-1751.

Wenzel, S.C., Gross, F., Zhang, Y., Fu, J., Stewart, A.F., and Müller, R. (2005). Heterologous expression of a myxobacterial natural products assembly line in pseudomonads via Red/ET recombineering. Chem Biol 12, 349-356.

Yu, D., Ellis, H.M., Lee, E.C., Jenkins, N.A., and Copeland, N.G. (2000). An efficient recombination system for chromosome engineering in Escherichia coli. Proc Natl Acad Sci USA 97, 5978-5983.

Zhang, Y., Muyrers, J.P., Testa, G., and Stewart, A.F. (2000). DNA cloning by homologous recombination in Escherichia coli. Nat Biotechnol $18,1314-1317$

Open Access This article is distributed under the terms of the Creative Commons Attribution License which permits any use, distribution, and reproduction in any medium, provided the original author(s) and source are credited. 